\title{
Towards Virtual H\&E Staining of Hyperspectral Lung Histology Images Using Conditional Generative Adversarial Networks
}

\author{
Neslihan Bayramog̃lu ${ }^{\S} \quad$ Mika Kaakinen * $\quad$ Lauri Eklund * Janne Heikkilä ${ }^{*}$ \\ $\S$ Center for Machine Vision and Signal Analysis, University of Oulu, Finland \\ * Faculty of Biochemistry and Molecular Medicine, University of Oulu, Finland \\ \{neslihan.bayramoglu, mika.kaakinen, lauri.eklund, janne.heikkila\}@oulu.fi
}

\begin{abstract}
The microscopic image of a specimen in the absence of staining appears colorless and textureless. Therefore, microscopic inspection of tissue requires chemical staining to create contrast. Hematoxylin and eosin $(H \& E)$ is the most widely used chemical staining technique in histopathology. However, such staining creates obstacles for automated image analysis systems. Due to different chemical formulations, different scanners, section thickness, and lab protocols, similar tissues can greatly differ in appearance. This huge variability is one of the main challenges in designing robust and resilient automated image analysis systems. Moreover, staining process is time consuming and its chemical effects deform structures of specimens.

In this work, we develop a method to virtually stain unstained specimens. Our method utilizes dimension reduction and conditional adversarial generative networks (cGANs) which build highly non-linear mappings between input and output images. Conditional GANs ability to handle very complex functions and high dimensional data enables transforming unstained hyperspectral tissue image to their $H \& E$ equivalent which comprises highly diversified appearance. In the long term, such virtual digital $H \& E$ staining could automate some of the tasks in the diagnostic pathology workflow which could be used to speed up the sample processing time, reduce costs, prevent adverse effects of chemical stains on tissue specimens, reduce observer variability, and increase objectivity in disease diagnosis.
\end{abstract}

\section{Introduction}

The examination of patient derived histological samples for pathological findings requires specialist expertise. However, a standard pathology laboratory may handle tens of samples daily which stands for the need of computer assisted image analysis and novel imaging technologies to

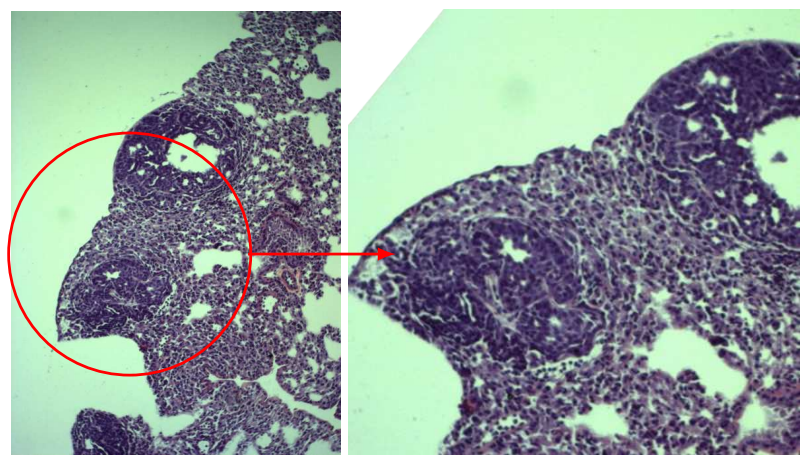

Figure 1. Hematoxylin and Eosin stained lung tissue section where cell nuclei are typically stained dark blue and cytoplasm pale blue/pink. (Right) Zoomed out version of the left image shows a metastatic foci where nuclear mass is typically higher giving the region a darker appearance, well distinguishable from nontransformed lung tissue. The same tissue section was also imaged using hyperspectral camera prior to staining (Figure 3).

help in prediagnosis. Routine sample processing includes fixation of a tissue sample and histological staining with hematoxylin and eosin $(\mathrm{H} \& \mathrm{E})$ to visualize tissue structures. However, appearance variability of H\&E stained sections creates major challenges in histopathology image analysis. These variations are due to variability among people, differences in protocols between labs, fixation, specimen orientation in the block, human skills in tissue preparation, microscopy maintenance, and color variation due to differences in staining procedures $[1,2]$. Still, pathologists prefer H\&E staining in the evaluation of many types of specimens to highlight or identify features that would provide important diagnostic information as they have been trained on similar cases and they understand these variations well. To limit the burden of time consuming sample processing and to aid in prediagnostics imaging of plain unstained tissue samples by capturing its intrinsic spectral information has proved promising $[3,4,5]$. Due to chemical effects of staining, transforming tissue undergoes changes in structure, molecular composition and quantities which reflect to 
changes in light scattering and absorption. Modern hyperspectral cameras are able to sample the spectral information in $\mathrm{nm}$ accuracy while simultaneously capturing 2D image of a specimen. Combining the spectral information with proof of concept virtual H\&E staining for verification is crucial for accurate diagnosis but in future hyperspectral imaging could even be used as a sole method for some diseases.

In this work, we develop a method to perform digital $\mathrm{H} \& \mathrm{E}$ staining on unstained hyperspectral microscopy image data, on a proof of concept level. The benefit of virtual H\&E staining in pathology would be following: i) avoiding time consuming sample processing, ii) getting rid of chemical effects on sample tissues, iii) cost savings, iv) eliminating visual variations due to different lab protocols, v) reduce observer variability, and vi) increase objectivity in disease diagnosis. Our method utilizes dimension reduction and conditional generative adversarial networks (cGANs) which build highly non-linear mappings between input and output images. Conditional GANs ability to handle very complex functions and high dimensional data enables transforming unstained hyperspectral tissue image to their $\mathrm{H} \& \mathrm{E}$ equivalent which comprises highly diversified appearance.

\section{Previous Work}

Hyperspectral imaging (HSI) is found to be beneficial in histopathology image analysis in several different studies [6]. Irshad et al. [7] used multispectral imaging for automated mitosis detection for breast cancer in histopathology images. They demonstrated the correlation between spectral bands and staining characteristics of H\&E. Zhou et al. [8] propose a multispectral unsupervised feature learning model for tissue classification, based on convolutional sparse coding and spatial pyramid matching. They showed that exploiting multiple spectra is beneficial for classification of histology sections which potentially contain diverse phenotypic signatures. Goto et al. [5] utilized hyperspectral imaging for differentiating gastric tumors from normal mucosa to establish a diagnostic algorithm. Kopriva et al. [9] employed blind-source separation to segment multispectral microscopic images of unstained specimens of human carcinoma. The main motivation in their work is to get rid of time consuming staining procedure. They nonlinearly map multichannel images into an image with an increased number of non-physical channels and a decreased correlation between spectral profiles. This mapping enables the separation of tissue components which could also be seen as a form of digital staining. Bautista and Yagi [10] present an approach to convert an H\&E stained multispectral image to its Massons trichrome stained equivalent by enhancement and linear transformation of spectral transmittance. It has been showed that the spectral features of H\&E stained tissue structures could be used to improve the visualization histopathology images. For detailed reviews on spec- tral imaging technology in biomedical engineering, we refer reader to the works of Li et al. [6] and Lu et al. [11].

In computational histopathology, previous attempts for digital staining approach the problem by finding a transformation equation that maps images from one domain to another domain usually in the least squares sense implemented by matrix manipulation [12,9]. Such approaches have limitation to produce reliable results as the input images are generally not standardized. Additionally, such mappings are not capable of handling very complex functions and high dimensional data to fully represent the relationship between source and target data.

To the best of authors knowledge, this is the first study which attempts to transform unstained hyperspectral images of tissues to their H\&E stained equivalent. In this work we develop a deep learning based method that performs virtual $H \& E$ staining of lung tissue sections by conditional generative adversarial networks.

\section{Virtual Staining}

The components of our virtual H\&E staining method is represented in Figure 2.

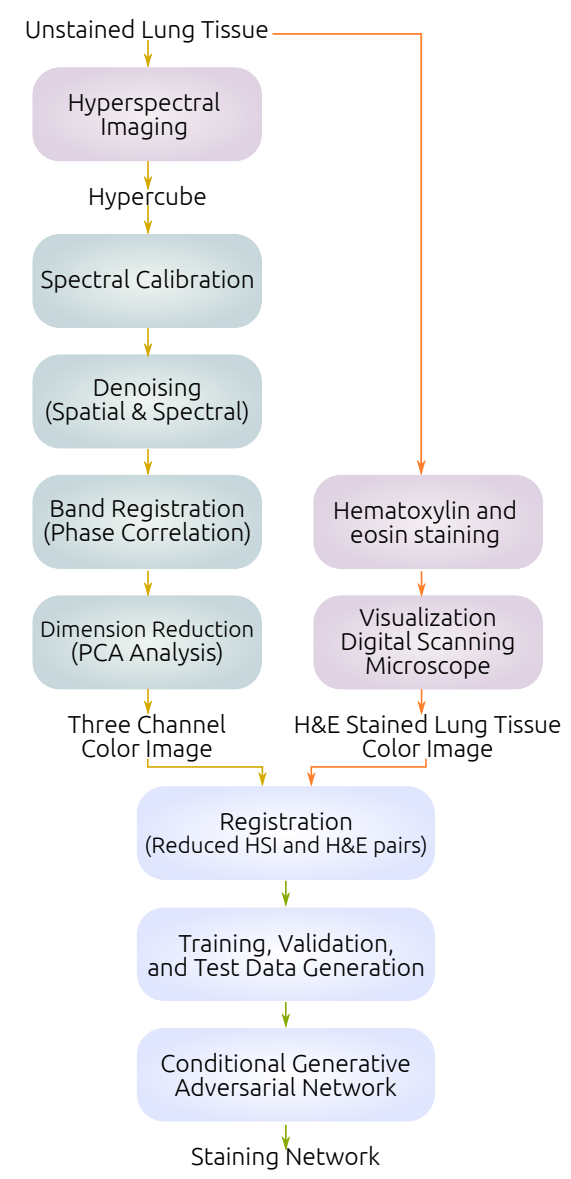

Figure 2. Flowchart of our virtual H\&E staining procedure for hyperspectral microscopy data. 


\subsection{Preprocessing Hyperspectral Data}

\subsubsection{Spectral Radiance Calibration}

In hyperspectral imaging systems, the raw data is sensitive to illumination and temperature. In order to calibrate raw images, both dark current image and a reference image are needed. Therefore, they are need to be taken just before sample acquisition. There is a current in hyperspectral image sensors even if the object under microscopy is not illuminated. This is called dark current and the hyperspectral data captured in this state is called dark current image. The reference image is captured to obtain the maximum reflectance in each wavelength which is performed with white glass material. The following formula is used in order to extract the actual spectral response of a sample in a pixel by pixel calibration fashion:

$$
I_{c a l \lambda, p}=\frac{S_{\lambda, p}-D_{\lambda, p}}{R_{\lambda, p}-D_{\lambda, p}} \quad \forall \lambda \text { and } p
$$

where $I_{c a l_{\lambda}, p}$ is the calibrated reflectance at pixel location $p$ for wavelength $\lambda . S_{\lambda, p}, D_{\lambda, p}$, and $R_{\lambda, p}$ are the sample, dark, and reference image values at the corresponding location and wavelength. Figure 3 shows a raw image after dark current normalization, reference image, and calibrated image at wavelength $500 \mathrm{~nm}$. Spectral calibration also corrects the artifacts resulted from the white glass impurities.

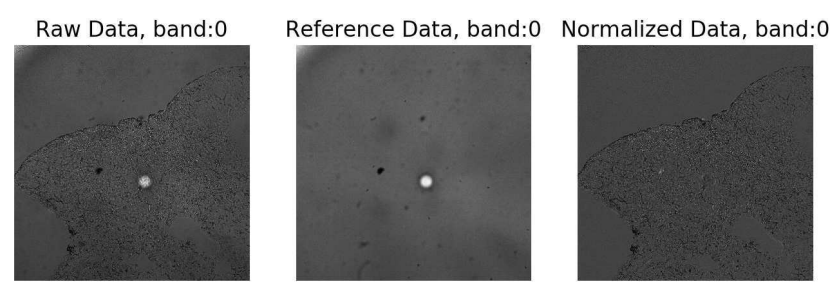

Figure 3. Spectral radiance calibration. (Left to right) Raw hyperspectral image after dark current normalization, reference data, and final calibrated data at wavelength $500 \mathrm{~nm}$.

\subsubsection{Band Registration}

Individual images in our hyperspectral cubes acquired by RikolaDT0030 ${ }^{1}$ camera are unaligned due to the motion of different sensors utilized for different bands of the light spectrum. This could be seen in Figure 4. However, having a proper band-by-band alignment is necessary for further analyses, particularly for the principal component analysis.

Phase Correlation We approach this alignment problem from image registration standpoint in the frequency domain. We adopt a translation motion model and estimate it based

\footnotetext{
${ }^{1}$ http://senop.fi/en/optronics-hyperspectral
}

on the shift property of the Fourier Transform using phase correlation. Given two images $f$ and $g$ related by a simple translation $(\Delta)$ and the corresponding Fourier Transforms are denoted $F(u)$ and $G(u)$ (i.e. $G(\mathbf{u})=e^{-2 \pi i \mathbf{u} \cdot \Delta} F(\mathbf{u})$ ). Then the normalized cross-power spectrum (phase correlation) is

$$
T(\mathbf{u}) \equiv \frac{F \cdot G^{*}}{|F| \cdot|G|}(\mathbf{u})=e^{2 \pi i \mathbf{u} \cdot \Delta}
$$

Then the Inverse Fourier Transform of cross-power spectrum gives a single peak. The location of the peak corresponds to the motion vectors between the two bands.

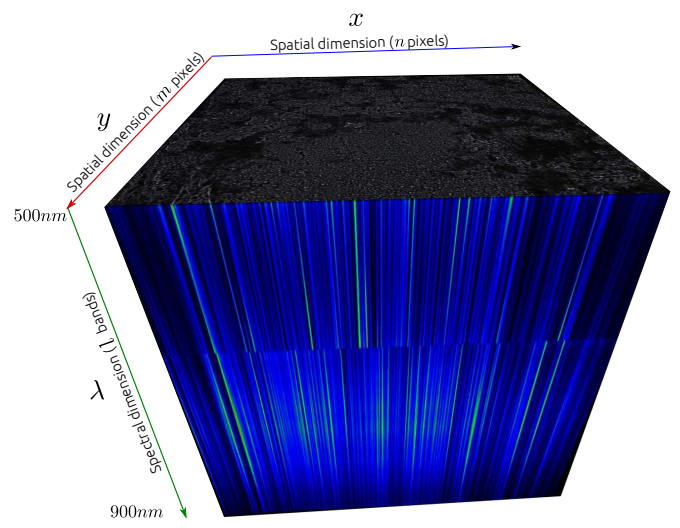

Figure 4. A sample hypercube acquired using RikolaDT0030. An obvious misalignment between wavelengths can be seen at the middle (at around wavelength $650 \mathrm{~nm}$ ).

\subsubsection{Denoising}

In order to remove the noise and enhance the hyperspectral information, we applied Gaussian filtering both in spectral and spatial dimension. The three dimensional Gaussian filter is defined by :

$$
g(x, y, \lambda)=\frac{1}{2 \pi \sqrt{2 \pi} \sigma_{x} \sigma_{y} \sigma_{\lambda}} \exp ^{-\left(\frac{x^{2}}{2 \sigma_{x}^{2}}+\frac{y^{2}}{2 \sigma_{y}^{2}}+\frac{\lambda^{2}}{2 \sigma_{\lambda}^{2}}\right)}
$$

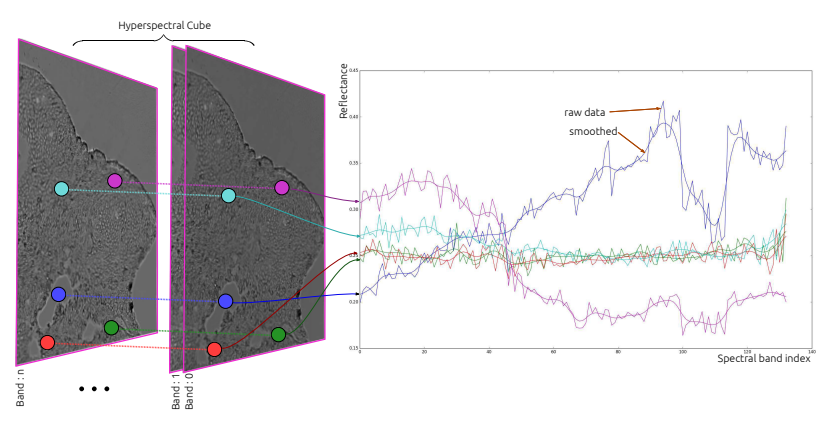

Figure 5. Spectral smoothing. 
where $\sigma_{x}, \sigma_{y}$, and $\sigma_{\lambda}$ are the standard deviations of the Gaussian filter among column, row and wavelength dimensions. Although Gaussian filtering smooths out details (i.e. sharp edges) in spatial domain by taking the weighted average (convolution) of the intensity of the adjacent pixels, it suppresses high frequency details like salt and pepper noise and spectral noise (Figure 5). In our case, spectral noise is higher than the spatial noise therefore, we applied bigger $\sigma$ along the spectral dimension.

\subsubsection{Dimension Reduction: Principal Component Analysis Transform}

The principal component analysis (PCA) is mainly used to reduce the dimension of hyperspectral images, which often contain redundant information as their adjacent bands are highly correlated. Therefore, most of the variance contained in the hyperspectral image can be represented using only a few principal components. While PCA removes the correlation among bands it also helps reducing the noise. Nevertheless, in our work flow we applied a denoising method prior to PCA transform. Lower dimensional data also beneficial for the computational complexity.

PCA examines the optimum linear combination of wavelengths to maximize the variation of pixel values in the least-square sense. Consider a hyperspectral data with $x$ rows, $y$ columns, and $m$ bands. Organize the hypercube into a $D=m \times n$ matrix where $n$ represents all the pixels of the image $(n=x \times y)$. A pixel vector can be defined as:

$$
p_{i}=\left[x_{1}, x_{2}, \ldots, x_{m}\right]_{i}^{T}, \quad i=1,2, \ldots n .
$$

One way to compute PCA is summarized as follows:

- Subtract the mean from the data

$$
\begin{gathered}
\mu=\frac{1}{n} \sum_{1}^{n} p_{i} \\
x_{i}=p_{i}-\mu
\end{gathered}
$$

- Compute the covariance matrix

$$
\operatorname{Cov}=\frac{1}{n} \sum_{1}^{n} x_{i} x_{i}^{T}
$$

- Compute eigenvalues and eigenvectors using singular value decomposition (SVD):

$$
D=U \Sigma U^{T}
$$

where $U=m \times m$ is a orthonormal matrix and $\Sigma$ is the diagonal singular value matrix composed of the eigenvalues $\lambda_{1}, \lambda_{2}, \ldots, \lambda_{m}$. The columns of $U$ are the eigenvectors of matrix $D$ and they are called principal components.
Projecting data onto new basis: Let the eigenvalues and eigenvectors are organized in descending order $\left(\lambda_{1} \geq \lambda_{2} \geq\right.$ $\left.\ldots \geq \lambda_{m}\right)$. The eigenvector $\left(e_{1}\right)$ that corresponds to the largest eigenvalue $\left(\lambda_{1}\right)$ of the covariance matrix shows the direction in the data with the highest variation. Similarly, the eigenvector that corresponds to the smallest eigenvalue contains the least variation in the hyperspectral image. The original data can be projected onto the new basis as follows:

$$
y_{i}=U^{T} p_{i}
$$

where $p_{i}$ is the original pixel vector (Equation 4) and $y_{i}$ is the projected pixel vector. First few components of the projected data contains most of the variance of the data. Therefore, dimension of a hyperspectral image can be reduced by selecting first $k$, where $k \ll m$, components of $y$ without loosing much information. The choice for selection of $k$ is usually based on the fraction of the energy (variation). In this study, we select $k=2$ as more than $95 \%$ of the signal variation is contained in the first two components. As a result, our hypercubes can be presented with three components: $i$ ) average image, $i$ i) first principal component, and iii) second principal component. This reduction enables us to represent a hyperspectral image as a single three channel color image (Figure 6).

\subsection{Preparing Dataset for Training}

Our staining network transforms unstained hyperspectral images, which are reduced to a three channel color image using PCA transform, to H\&E stained like images. During the training phase, the network needs registered image pairs of unstained and stained versions of the same tissue. Following the hyperspectral imaging, lung tissue slides undergo a staining procedure. Consequently, unstained hyperspectral imaging and microscopy imaging of H\&E stained sections are performed at different imaging systems, therefore, they are not registered. Their resolution, orientations, and field of views are different (See Figure 1 and Figure 3). Moreover, chemical staining procedure slightly deforms tissues (local shrinking or enlargement).

Registration of $\mathrm{H \& E}$ stained images with $\mathrm{PCA}$ reduced hyperspectral data: We model the geometric transformation between reduced HSI and H\&E image as a perspective transformation and perform the registration using fiducial points. Since images differ by elastic deformations, we manually select corresponding points in these images (Figure 7). The problem is to find a mapping $H$ (homography matrix) to map pixel locations $(q)$ in source image to locations $\left(q^{\prime}\right)$ in the destination image using homogeneous coordinates:

$$
q^{\prime}=H q, \quad \lambda\left(\begin{array}{l}
x^{\prime} \\
y^{\prime} \\
1
\end{array}\right)=\left(\begin{array}{lll}
H_{11} & H_{12} & H_{13} \\
H_{21} & H_{22} & H_{23} \\
H_{31} & H_{32} & H_{33}
\end{array}\right)\left(\begin{array}{l}
x \\
y \\
1
\end{array}\right) .
$$



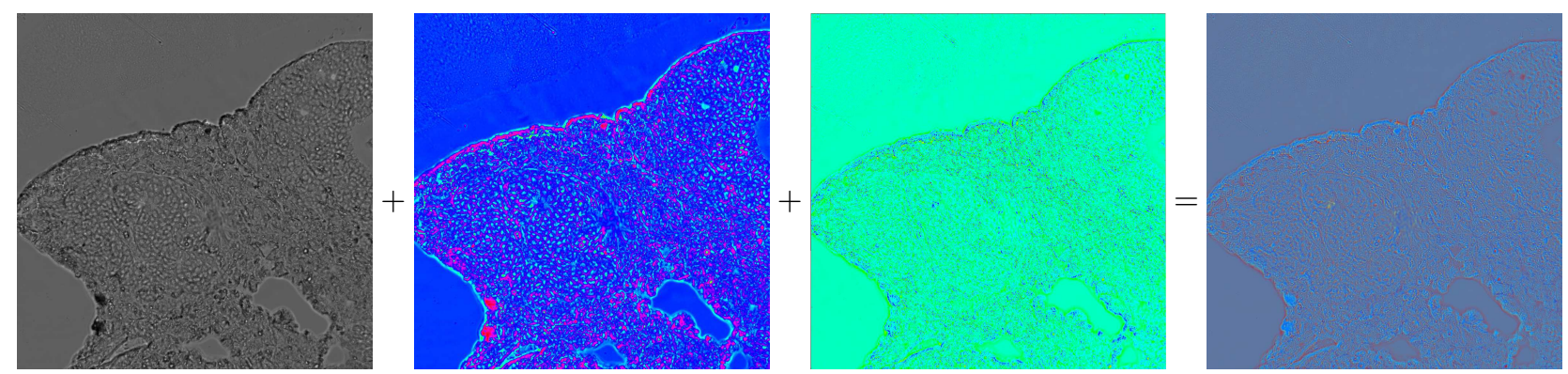

Figure 6. Reduced hyperspectral image data. We used average image and the first two components of the PCA transformation to represent a hypercube which leads to a three channel color image. For visualization purposes, principal components are shown in HSV colormap.
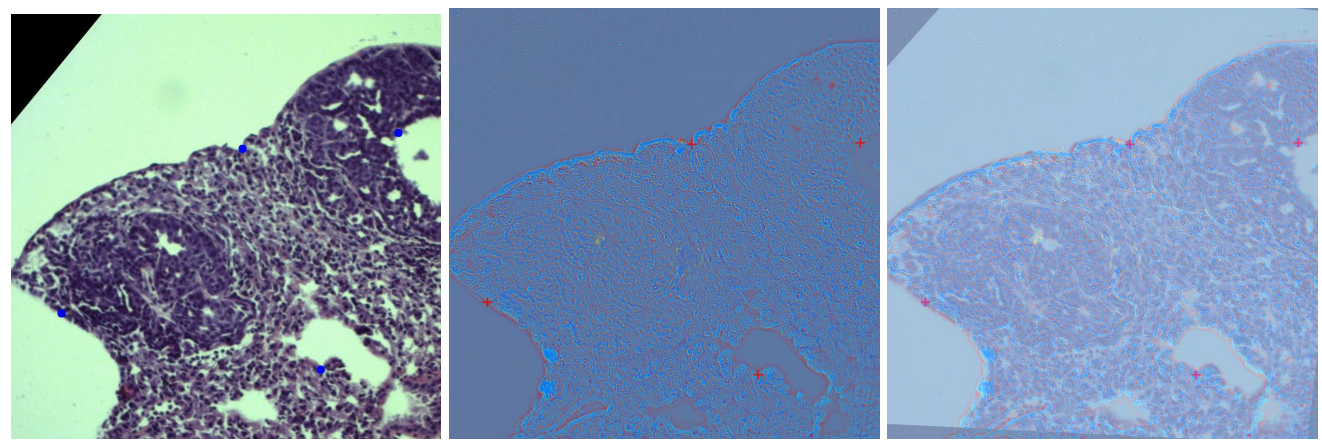

Figure 7. H\&E stained images and HSI images differ in resolution, orientations, and field of view. Local elastic deformations are also introduced in H\&E images due to chemical staining. We manually select four fiducial points in both modalities to perform the registration. (Left) H\&E stained slide subsection with manually marked four (blue) points. (Middle) Reduced HSI of the same slide with manually marked four (red) points. (Right) Registered image pairs (overlaid).

Matrix $\mathrm{H}$ can be computed using 4 points with a simple least squares scheme. If there are more than 4 points with outliers, then the inliers are calculated using RANSAC method and the homography matrix is then refined using Levenberg-Marquardt method [13].

Both imaging modalities (HSI and bright field microscopy) produce large images reaching to size about $1000 \times 1000$ pixels; therefore, after the registration, we cropped images into smaller patches to create training image pairs. In this way, both computational complexity can be reduced and a smaller neural network can be trained which requires fewer training samples due to smaller number of parameters. Learning stage of our neural network is not hampered because cells and other biological structures are much smaller than our patch size (see Figure 9).

\subsection{Conditional Generative Adversarial Networks}

We learn a mapping from reduced HSI to H\&E stained image using conditional generative adversarial network (cGAN) [14] which is an extension of generative adversarial networks (GANs)[15].

GANs are introduced in 2014 by Goodfellow et al. [15] to model the training image data distribution which is then used to generate new image samples from the same distribution. They consist of two networks: generator $(G)$ and dis- criminator $(D)$. The generative model $G$ learns a mapping from training data to generate new samples from some prior distribution (random noise vector) by imitating the real data distribution. On the other hand, the discriminator $D$ tries to classify images generated by $G$ whether they came from real training data (true distribution) or fake. These two networks are trained at the same time and updated as if they are playing a game. That is, generator $G$ tries to fool discriminator $D$ and in turn discriminator $D$ adjust its parameters to make better estimates to detect fake images generated by $G$.

Conditional GANs are extensions of GANs where both generator and discriminator are conditioned on additional information. They are used to transform images from one image domain to another image domain when the auxiliary information is image [14, 16] Adversarial learning in cGANs is achieved by minimizing the following objective cost function:

$$
\begin{aligned}
\mathcal{L}_{c G A N}(G, D) & =\mathbb{E}_{x, y \sim p_{\text {data }}(x, y)}[\log D(x, y)] \\
& +\mathbb{E}_{x \sim p_{\text {data }}(x)}[\log (1-D(x, G(x))] \\
& +\lambda \mathbb{E}_{x, y \sim p_{\text {data }}(x, y)}\left(\|y-G(x)\|_{1}\right),
\end{aligned}
$$

where $x$ is the input reduced HSI, $y$ is the corresponding $\mathrm{H} \& \mathrm{E}$ stained image, $p_{\text {data }}(x)$ is the real image distribution 
in the training set, $p_{\text {data }}(x, y)$ is the joint probability distribution of input and output image pairs, and $\mathbb{E}_{x, y \sim p_{\text {data }}(x, y)}$ is the expectation of log likelihood of $(x, y)$ (Figure 8). Global $L_{1}$ loss (last term in Equation 11) is proposed by Isola et al.[14] to reduce blurring and generate sharper images. The $\lambda$ component balances the adversarial loss and global loss.

We follow Isola et al. [14] and utilize "U-Net" [17] based architecture for the generator $G$ which includes skip connections between each layer $i$ and layer $n-i$, where $n$ is the total number of layers. Similarly, we used convolutional "ImageGAN" classifier [18] for the discriminator network $D$. Both network architectures are composed of layers of Convolution-BatchNorm-ReLu or ConvolutionBatchNorm-Dropout-ReLu modules. For details, we refer reader to Isola et al. [14].

\section{Experiments and Results}

Lung tissue samples were obtained from PyMT (VB/NTg(MMTV-PyMT)634Mul/J mouse stain which spontaneously develops breast carcinoma. The tumors progress to metastasis in the lungs at relatively early stage of tumorigenesis. The lungs excised from euthanized animals were snapfrozen in liquid nitrogen and sectioned in $10 \mathrm{~m}$ sections with cryomicrotome (Leica CM3050S ). The sections were immediately imaged with Rikola Hyperspectral Imager ( Senop ltd.) capable for acquiring snapshots with $1010 \times 1010$ pixel resolution, each pixel covering 500-1000 $\mathrm{nm}$ spectral range. The camera was connected to Zeiss Upright microscope (AX10) and sample illuminated with halogen lamp (voltage set to maximum). Objectives 10x (NA $0,3)$ or 20x (NA 0,5) were used. After acquiring HSI images the sections were fixed with $4 \%$ paraformaldehyde in phosphate buffered saline and stained with Harris hematoxylin and eosin followed by series of dehydration steps with in-

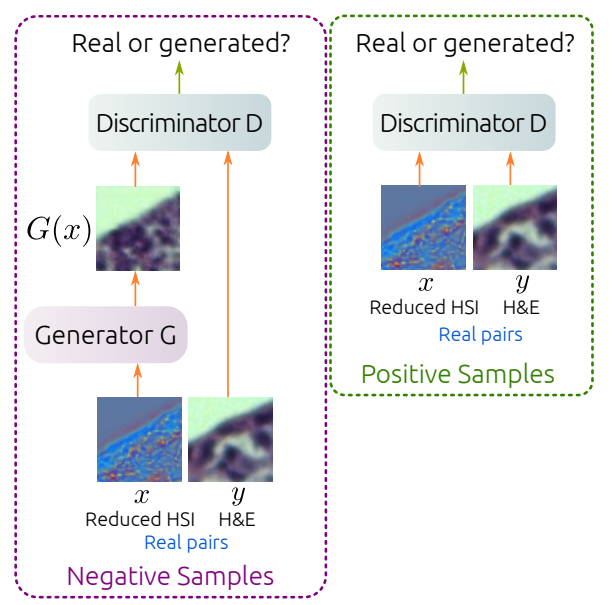

Figure 8. Graphical overview of conditional GAN training.

\begin{tabular}{|l||c|c|}
\hline Dataset $\backslash$ Method & SSIM & MSE \\
\hline \hline Test & 0.3873 & $2.44 E 3$ \\
Validation & 0.3844 & $2.47 E 3$ \\
\hline
\end{tabular}

Table 1. Averaged SSIM and MSE of our staining network

creasing concentrations of ethanol. The images from H\&E stained sections, corresponding the regions captured with hyperspectral camera, were acquired with Leicas DFC320 color camera.

Our hyperspectral image cubes are composed of 133 bands. We reduce the dimension of our hypercubes to 3 via PCA transform. Finally, the input (reduced HSI) and output (virtual H\&E staining) of our staining network are three channel images. The registered HSI and H\&E image pairs are divided into overlapping patches of $64 \times 64$ pixels with a stride of 16 pixels. As a result, we obtain 1418 training image pairs, 994 test image pairs, and 426 image pairs for validation. Dataset and codes are available at http://www.ee.oulu.fi/ nyalcinb.

Generator and discriminator networks are trained from scratch and weights are initialized from a Gaussian distribution with 0 mean and 0.2 standard deviation. A dropout rate of 0.5 is used in our experiments. We trained our staining network model for 100 epochs until discriminator loss of validation rises and generator loss stabilizes. For optimizing our networks we used mini batch stochastic gradient descent (SGD) with Adam solver and alternate one step gradient between $D$ and $G$. Experiments are performed on Nvidia GTX 750 Ti GPU. Due to lack of computation power we utilize a mini batch size of 20 images. Figure 11 shows loss of the discriminator and generator networks. As generator loss decreases discriminator loss tends to rise to the maximal ambiguity (0.5) which is a good indicator that they challenge each other.

In Figure 9, we present qualitative results generated by our staining network from the test set. The generated images are visually similar to real images but they also have clear differences. Due to local deformations, which is clearly seen in the test sample 4 , synthesized images do not fully overlap with ground truth, they follow the structure of reduced HSI. Quantitative evaluation of generative models is hard and a challenging problem [14]. We adopt the Structural Similarity (SSIM) index [19] to measure the similarity between synthesized images by our staining network and ground truth image (Table 1). SSIM between two images $x$ and $y$ is defined as follows:

$$
\operatorname{SSIM}(x, y)=\frac{\left(2 \mu_{x} \mu_{y}+C_{1}\right)\left(2 \sigma_{x y}+C_{2}\right)}{\left(\mu_{x}^{2}+\mu_{y}^{2}+C_{1}\right)\left(\sigma_{x}^{2}+\sigma_{y}^{2}+C_{2}\right)}
$$

where $\mu$ is the average image, $\sigma$ is the standard deviation, $\sigma_{x y}$ is the cross covariance of images $x$ and $y$, and $C$ 's are constants to avoid division by zero. SSIM values are in the 


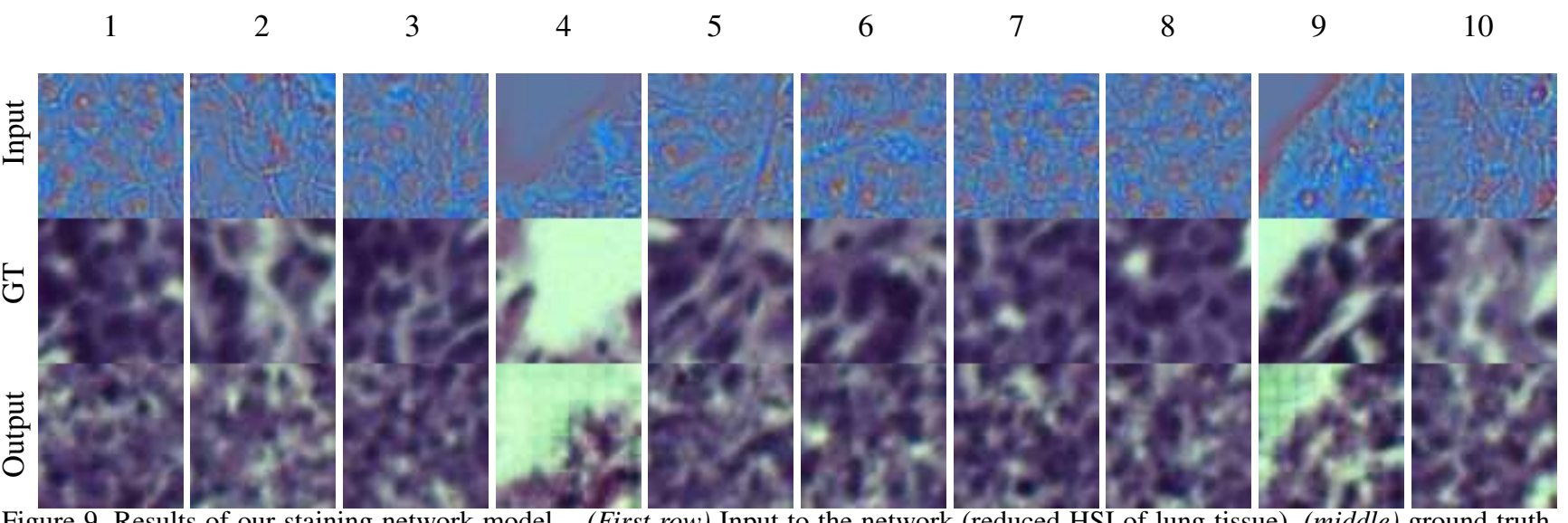

Figure 9. Results of our staining network model. (First row) Input to the network (reduced HSI of lung tissue), (middle) ground truth, (bottom) generated images using our staining network model.
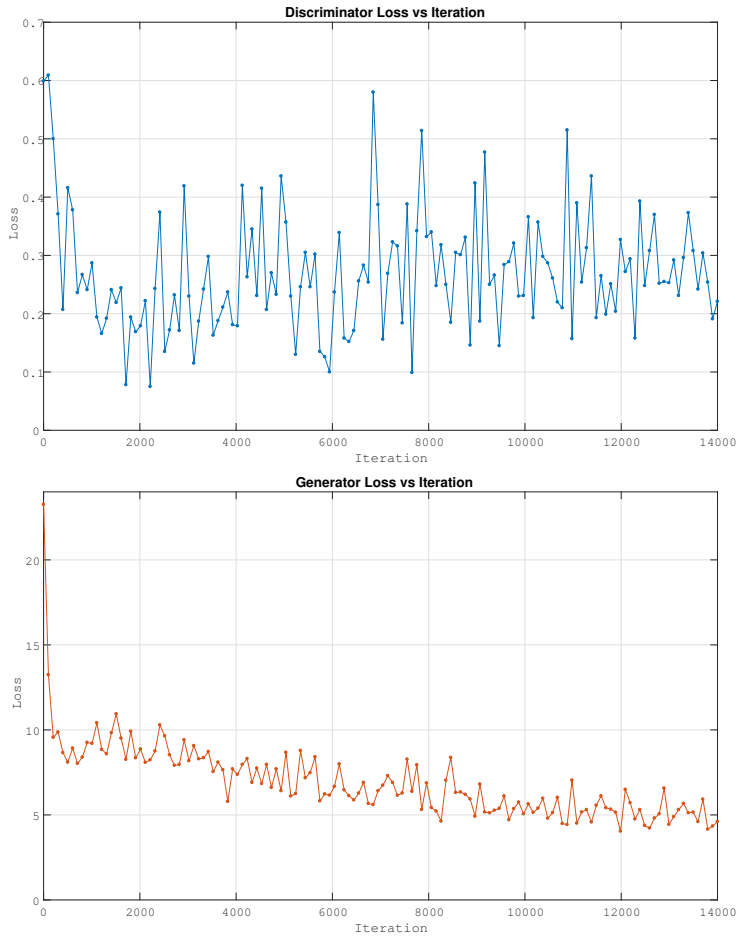

Figure 10. Discriminator and generator loss during training.

range of $[-1,1]$, where higher values indicate more structural similarity. The color version of SSIM utilize averaging SSIMs for each channel. We also present mean squared pixel-wise error (MSE) in Table 1. However, both quantitative evaluation (SSIM, MSE) and subjective visual evaluation does not answer if our system generates realistic staining or not. They are not fully correlated with human perception. In our case, the golden standard could only be obtained from pathologist views which we plan to do it as a future work. However, this is usually subjective and timeconsuming. The golden standard would be more reliable if the feedbacks from multiple pathology experts could be combined.

\section{Conclusion and Future Work}

In diagnostic pathology, tissue samples undergo a series of processes. Tissue staining is one of the critical step in this pipeline. It enables us to see tissues under light microscope which are almost visible without stains. Hematoxylin and eosin is the most widely used chemical staining technique in histology. However, such staining creates obstacles for automated image analysis systems. Due to different chemical formulations, different scanners, section thickness, and lab protocols, similar tissues can greatly differ in appearance. This huge variability is one of the main challenges in designing robust and resilient automated image analysis systems.

In this paper, we developed a system which uses conditional generative adversarial networks to virtually stain unstained hyperspectral lung tissue histopathology images to look like their H\&E stained versions. Our trained staining network model provides a transformation from hyperspectral domain to H\&E domain. Our results are promising for producing $\mathrm{H} \& \mathrm{E}$ stained tissues virtually. However, it needs to be validated by pathologists and the value for its clinical use could only be evaluated afterwards. As a future work, we plan to evaluate the results by collecting pathologists' feedback. Feedbacks could simply be a similarity score between generated and ground truth images. Considering the subjective evaluation and variation among experts, we plan to ask several pathologist for evaluation. Moreover, we would like to explore the deterministic effects of our condition set, network components, and loss function on the output image. If it gets clinically accepted, virtual histology staining would facilitate the workload of the diagnostic pathology by eliminating the visual variabilities among tissue samples to produce objective diagnosis, skipping man- 

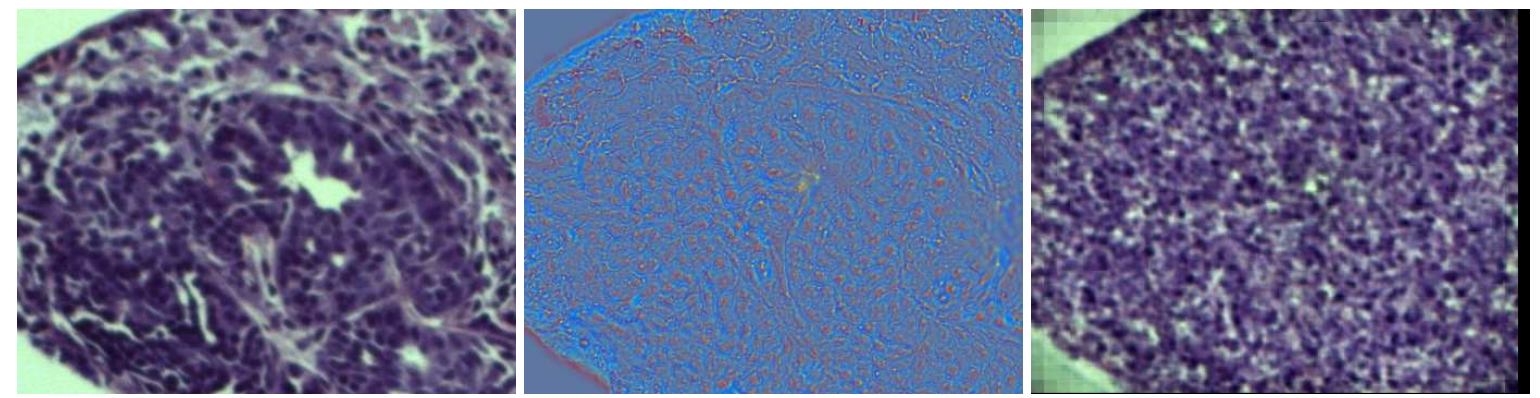

Figure 11. (Left) Real H\&E stained image. (Middle) Corresponding reduced HSI, input to the network. (Right) Synthesized image with our staining network. Generated test patches are overlaid to visualize the final composition.

ual staining work, and consequently reducing the amount of time for obtaining digital images. In addition, staining and tissue preparation can be inconsistent within a sample. This technique could potentially be used to detect areas where the specimen has not been stained very well and so provide an overall confidence indicator for the H\& E staining based on the HSI.

\section{References}

[1] M. T. McCann, J. A. Ozolek, C. A. Castro, B. Parvin, and J. Kovacevic, "Automated histology analysis: Opportunities for signal processing," IEEE Signal Processing Magazine, vol. 32, no. 1, pp. 78-87, 2015. 1

[2] N. Bayramoglu, J. Kannala, and J. Heikkilä, “Deep learning for magnification independent breast cancer histopathology image classification," in ICPR, pp. 2440-2445, IEEE, 2016. 1

[3] D. G. Ferris, R. A. Lawhead, E. D. Dickman, N. Holtzapple, J. A. Miller, S. Grogan, S. Bambot, A. Agrawal, and M. L. Faupel, "Multimodal hyperspectral imaging for the noninvasive diagnosis of cervical neoplasia," Journal of Lower Genital Tract Disease, vol. 5, no. 2, pp. 65-72, 2001. 1

[4] M. C. Pierce, R. A. Schwarz, V. S. Bhattar, S. Mondrik, M. D. Williams, J. J. Lee, R. Richards-Kortum, and A. M. Gillenwater, "Accuracy of in vivo multimodal optical imaging for detection of oral neoplasia," Cancer Prevention Research, vol. 5, no. 6, pp. 801-809, 2012. 1

[5] A. Goto, J. Nishikawa, S. Kiyotoki, M. Nakamura, J. Nishimura, T. Okamoto, H. Ogihara, Y. Fujita, Y. Hamamoto, and I. Sakaida, "Use of hyperspectral imaging technology to develop a diagnostic support system for gastric cancer," Journal of biomedical optics, vol. 20, no. 1, pp. 016017-016017, 2015. 1, 2

[6] Q. Li, X. He, Y. Wang, H. Liu, D. Xu, and F. Guo, "Review of spectral imaging technology in biomedical engineering: achievements and challenges," Journal of biomedical optics, vol. 18, no. 10, pp. 100901-100901, 2013. 2

[7] H. Irshad, A. Gouaillard, L. Roux, and D. Racoceanu, "Spectral band selection for mitosis detection in histopathology," in Biomedical Imaging (ISBI), 2014 IEEE 11th International Symposium on, pp. 1279-1282, IEEE, 2014. 2
[8] Y. Zhou, H. Chang, K. Barner, P. Spellman, and B. Parvin, "Classification of histology sections via multispectral convolutional sparse coding," in Proceedings of the IEEE CVPR, pp. 3081-3088, 2014. 2

[9] I. Kopriva, M. P. Hadžija, M. Hadžija, and G. Aralica, "Unsupervised segmentation of low-contrast multichannel images: discrimination of tissue components in microscopic images of unstained specimens," Scientific reports, vol. 5, 2015. 2

[10] P. A. Bautista and Y. Yagi, "Digital simulation of staining in histopathology multispectral images: enhancement and linear transformation of spectral transmittance," Jnl. of biomedical optics, vol. 17, no. 5, pp. 0560131-05601310, 2012. 2

[11] G. Lu and B. Fei, "Medical hyperspectral imaging: a review," Journal of biomedical optics, vol. 19, 2014. 2

[12] P. A. Bautista, T. Abe, M. Yamaguchi, Y. Yagi, and N. Ohyama, "Digital staining of unstained pathological tissue samples through spectral transmittance classification," Optical review, vol. 12, no. 1, pp. 7-14, 2005. 2

[13] R. Szeliski, Computer vision: algorithms and applications. Springer Science \& Business Media, 2010. 5

[14] P. Isola, J.-Y. Zhu, T. Zhou, and A. A. Efros, "Imageto-image translation with conditional adversarial networks," arXiv preprint arXiv:1611.07004, 2016. 5, 6

[15] I. Goodfellow, J. Pouget-Abadie, M. Mirza, B. Xu, D. Warde-Farley, S. Ozair, A. Courville, and Y. Bengio, "Generative adversarial nets," in Advances in neural information processing systems, pp. 2672-2680, 2014. 5

[16] P. Costa, A. Galdran, M. I. Meyer, M. D. Abràmoff, M. Niemeijer, A. M. Mendonça, and A. Campilho, "Towards adversarial retinal image synthesis," arXiv preprint arXiv:1701.08974, 2017. 5

[17] O. Ronneberger, P. Fischer, and T. Brox, "U-net: Convolutional networks for biomedical image segmentation," in MICCAI, pp. 234-241, Springer, 2015. 6

[18] C. Li and M. Wand, "Precomputed real-time texture synthesis with markovian generative adversarial networks," in European Conference on Computer Vision, pp. 702-716, Springer, 2016. 6

[19] Z. Wang, A. C. Bovik, H. R. Sheikh, and E. P. Simoncelli, "Image quality assessment: from error visibility to structural similarity," IEEE, TIP, vol. 13, no. 4, pp. 600-612, 2004. 6 